\title{
Influência do butorfanol sobre os períodos de latência e de ação da ropivacaína pela via peridural na ovariossalpingo-histerectomia em cadelas
}

\author{
[Influence of butorphanol on latency and duration of ropivacaine epidural anesthesia for \\ ovariosalpingohysterectomy in bitches] \\ V.B. Albuquerque ${ }^{1}$, T.F.B. Souza ${ }^{2}$, M.C.R. Vivan ${ }^{2}$, J.Z. Ferreira ${ }^{2}$, M.C. Frade ${ }^{3}$, \\ S.H.V. Perri ${ }^{4}$, V.N.L.S. Oliva ${ }^{5 *}$ \\ ${ }^{1}$ Aluna de pós-graduação - FMB-UNESP - Botucatu, SP \\ ${ }^{2}$ Aluna de pós-graduação - UNESP - Araçatuba, SP \\ ${ }^{3}$ Médico veterinário autônomo \\ ${ }^{4}$ Departamento de Apoio, Produção e Saúde Animal - UNESP - Araçatuba, SP \\ ${ }^{5}$ Departamento de Clínica, Cirurgia e Reprodução Animal - UNESP \\ Rua Clóvis Pestana, 793 \\ 16050-680 - Araçatuba, SP
}

\begin{abstract}
RESUMO
\end{abstract}
Avaliaram-se os períodos de latência e de duração do efeito do butorfanol associado à ropivacaína aplicados pela via peridural e a possibilidade de uso dessa associação como protocolo anestésico para realização de ovariossalpingo-histerectomia (OSH) em cadelas. Utilizaram-se 16 cadelas pré-medicadas com acepromazina e midazolam compondo dois grupos: no grupo $1(\mathrm{n}=8)$ aplicou-se ropivacaína isolada $(0,3 \mathrm{~mL} / \mathrm{kg})$ e no grupo 2 $(\mathrm{n}=8)$, butorfanol $(0,1 \mathrm{mg} / \mathrm{kg})$ e ropivacaína (até o volume de $0,3 \mathrm{~mL} / \mathrm{kg}$ ), pela via peridural. Consideraram-se nove momentos $(\mathrm{M}): \mathrm{M}_{0}$ - animal sem anestesia, $\mathrm{M}_{1}-15$ minutos após medicação pré-anestésica; $\mathrm{M}_{2}-30$ minutos após a aplicação peridural; $\mathrm{M}_{3}, \mathrm{M}_{4}$ e $\mathrm{M}_{5}$ - correspondentes ao início do procedimento e ao pinçamento dos pedículos esquerdo e direito, respectivamente; $\mathrm{M}_{6}$ - ligadura da cérvix uterina; e $\mathrm{M}_{7}$ e $\mathrm{M}_{8}$ - início da laparorrafia e fim da sutura de pele, respectivamente. Foram avaliados os períodos de latência e ação do bloqueio, além da viabilidade de realização do procedimento cirúrgico por meio do bloqueio efetuado. O que recebeu ropivacaína + butorfanol apresentou sedação pronunciada e permitiu a realização de OSH em $75 \%$ dos animais, sem indução anestésica. Não houve diferença entre os grupos quanto aos períodos de latência e duração. A associação do butorfanol à ropivacaína proporcionou bloqueio anestésico compatível com a realização de OSH e período de latência curto, com duração de efeito suficiente para o procedimento cirúrgico.

Palavras-chave: cadela, butorfanol, ropivacaína, peridural

\begin{abstract}
The periods of latency and duration of butorphanol associated with ropivacaine used via epidural, and this combination as anesthetic protocol for carrying out ovariosalpingohysterectomy $(\mathrm{OSH})$ in bitches were evaluated. Sixteen animals pre-medicated with acepromazine and midazolam were used composing two groups that received: $1(n=8)$ ropivacaine $(0.3 \mathrm{~mL} / \mathrm{kg})$ and $2(n=8)$ butorphanol $(0.1 \mathrm{mg} / \mathrm{kg}$ ) and ropivacaine (up to the volume of $0.3 \mathrm{ml} / \mathrm{kg}$ ) via epidural. Nine moments were studied: $M_{1}-15$ minutes after pre-anesthetic medication; $M_{2}-30$ minutes after the epidural medication; $M_{3}, M_{4}$, and $M_{5}-$ at the beginning of surgery and at clamping left and right pedicles, respectively; $M_{6}-$ at ligation of the uterine cervix; and $M_{7}$ and $M_{8}-a t$ laparorhaphy and end of skin suture, respectively. Periods of latency and blocking the action of random double-covert manner were evaluated as well as the feasibility of carrying out the surgery performed by the blockade. It was observed that the group receiving ropivacaine + butorphanol allowed the execution of OSH in $75 \%$ of animals without the need for anesthesia. There was no statistical difference between the periods of latency and duration. It was concluded that the combination of butorphanol to ropivacaine provides, in bitches, block compatible with the implementation of $\mathrm{OSH}$ with short period of latency and duration of effect sufficient for the surgical procedure.
\end{abstract}

Keywords: bitches, butorphanol, ropivacaine, epidural

Recebido em 28 de fevereiro de 2009

Aceito em 12 de julho de 2010

*Autor para correspondência (corresponding author)

E-mail: voliva@fmva.unesp.br 


\section{INTRODUÇÃO}

A anestesiologia veterinária busca, a cada dia protocolos mais seguros, versáteis e com menos efeitos colaterais, a fim de melhor atender às necessidades dos pacientes e da equipe cirúrgica. Vários aspectos positivos têm sido considerados com o uso da anestesia epidural lombossacra para realização de ovariossalpingo-histerectomia $(\mathrm{OSH})$ em cadelas, destacando-se mínimas alterações cardiorrespiratórias, controle da dor pós-operatória, além da redução do estresse trans-operatório. A altura do bloqueio alcançado por essa via depende de vários fatores, destacando-se o tipo e o volume do fármaco utilizado.

Carvalho e Luna (2007) relataram que a região responsável pela inervação dos ovários pode ser bloqueada com doses altas de anestésico local ou pela associação deste a outros fármacos anestésicos. Contudo, Gasparini et al. (2007), ao utilizarem a ropivacaína ou lidocaína isoladas ou a associação da lidocaína à xilazina pela via peridural, constataram que a associação do agonista alfa-2 promoveu progressão mais cranial do bloqueio, viabilizando o procedimento cirúrgico de $\mathrm{OSH}$.

A utilização de opioides pela via peridural tem alcançado grande ênfase nos últimos anos (Fantoni, 2000), e resultados satisfatórios têm sido obtidos com a associação desses aos anestésicos locais em cirurgias pré-umbilicais (Cassu et al., 2005). Ishiy et al. (2002) observaram que a extensão cranial do bloqueio anestésico chegou até $\mathrm{T}_{12}$ ao associarem lidocaína e butorfanol pela via peridural, o que permitiu a realização de cirurgia de $\mathrm{OSH}$ em cadelas.

A ropivacaína é um anestésico local que possui um grupamento amino-amida e promove ação de longa duração (Alahuhta et al., 1995). Possui propriedades vasoconstrictoras intermediárias, não necessita da adição de adrenalina (Andrade et al., 2002) e é, ainda, cerca de três a quatro vezes mais potente que a lidocaína e de efeito mais prolongado. Freitas et al. (2004) demonstraram que a ropivacaína a $0,75 \%$ é a mais indicada para bloqueios anestésicos por ser menos cardiotóxica do que a bupivacaína, e Plowman et al. (1998) também relataram menor potencial de toxicidade no sistema nervoso central em relação à bupivacaína em humanos e em animais. Segundo Etches et al. (1997), a ropivacaína promove bloqueio sensitivo de duração igual ou pouco menor do que a bupivacaína e período de bloqueio motor mais curto.

O butorfanol é um opioide agonista sintético dos receptores kappa $(\kappa)$ e um fraco antagonista dos receptores mi $(\mu)$. Seu efeito analgésico ocorre na subcortical e espinhal, sendo, portanto, melhor modulador de analgesia visceral do que somática (Webster, 2005). Segundo Luna (1999), o butorfanol, quando utilizado por via epidural, promove analgesia de longa duração e sem depressão do sistema nervoso central. Tsang et al. (1998), em ratos, constataram que a infusão intratecal de butorfanol produzia analgesia visceral dose-dependente e de maneira eficaz, promovendo eficiente alívio da dor crônica. Entretanto, desenvolve alguns episódios de tolerância.

Houghton et al. (1991) afirmaram que o butorfanol pode ser utilizado de maneira isolada, promovendo analgesia visceral com duração de cerca de 30 minutos e sedação que pode chegar a 75 minutos, na dose de $0,4 \mathrm{mg} / \mathrm{kg}$, pela via intravenosa em cães. Ishiy et al. (2002), ao associarem lidocaína ao butorfanol via peridural, constataram um aumento do tempo de duração do bloqueio em comparação ao uso do anestésico local isolado. Maia (2006), ao utilizar o butorfanol em associação com a ropivacaína pela via peridural, obteve um grau de sedação mais intenso, comparado à sedação de grupos em que a ropivacaína foi associada à petidina ou à morfina, em cães.

Sendo assim, o presente estudo teve por objetivos determinar o período de latência e o período de duração do efeito da associação do butorfanol à ropivacaína pela via peridural e avaliar seus efeitos sistêmicos em cadelas.

\section{MATERIAL E MÉTODOS}

O estudo foi realizado com 16 cadelas hígidas, adultas, de diferentes raças, com pesos entre 6 e $18 \mathrm{~kg}$, triadas junto à comunidade local e préselecionadas para realização de OSH eletiva. Os animais foram submetidos à coleta de sangue para realização de hemograma completo, à sorologia para detecção de leishmaniose (por 
tratar-se de uma região endêmica para a doença) e a exames físicos para excluir os que apresentassem alguma alteração. Para a realização do procedimento anestésico-cirúrgico, as cadelas, em jejum alimentar de 12 horas e hídrico de quatro horas, foram submetidas à medicação pré-anestésica (MPA), que constou da associação de acepromazina $(0,05 \mathrm{mg} / \mathrm{kg})$ (Acepran 1\% - Univet S/A Ind. Veterinária ) e midazolam $(0,2 \mathrm{mg} / \mathrm{kg})$ (Dorrmire - Cristália prod. Químicos e farmacêuticos Ltda.) pela via intramuscular, realizando-se, na sequência, a aplicação de $0,5 \mathrm{~mL}$ de ropivacaína via subcutânea (botão anestésico) no local de colocação posterior da agulha para o bloqueio peridural. Decorridos 15 minutos, realizou-se a anestesia peridural na região lombossacra $\left(\mathrm{L}_{7}-\right.$ $\mathrm{S}_{1}$ ), após prévia tricotomia e antissepsia. Para tanto, posicionou-se o animal em decúbito esternal com os membros pélvicos estendidos cranialmente e introduziu-se uma agulha hipodérmica $40 \quad$ x $8 \mathrm{~mm}$ ou um cateter (dependendo do peso e tamanho do animal) no espaço intervertebral até ultrapassar o ligamento amarelo e atingir o espaço peridural. Confirmava-se a localização correta da agulha pela observação da aspiração de uma gota de solução de $\mathrm{NaCl}$ a $0,9 \%$ colocada previamente no canhão da agulha.

Os animais foram alocados em dois grupos distintos: os do grupo $1(\mathrm{n}=8)$ receberam ropivacaína (Naropin $0,75 \%$ - Astra-Zeneca do Brasil Ltda.) $(0,3 \mathrm{~mL} / \mathrm{kg})$ e os do grupo $2(\mathrm{n}=8)$, ropivacaína + butorfanol (Torbugesic - Fort Dodge Animal Health ) $(0,1 \mathrm{mg} / \mathrm{kg}$ completandose o volume até $0,3 \mathrm{~mL} / \mathrm{kg}$ com o anestésico local). Determinou-se a ordem de participação em cada grupo de maneira aleatória e duplamente encoberta tanto para o cirurgião como para o anestesista (que foram sempre os mesmos durante todo o estudo para não haver influência nos resultados). Após a aplicação da anestesia peridural (período de infusão padronizado $=1$ minuto), mantinham-se os animais em decúbito esternal por 30 minutos, a fim de que houvesse distribuição bilateral dos fármacos.

Era então avaliada a possibilidade de realização do procedimento cirúrgico mediante a tranquilização e o bloqueio ocorridos. Caso houvesse manifestação de dor durante a cirurgia, indicada pela elevação significativa da frequência (FC) ou da pressão arterial sistólica (PAS) ou mesmo pela movimentação do animal frente ao estímulo doloroso, realizava-se a indução anestésica com tiopental $(6,5 \mathrm{mg} / \mathrm{kg})+$ midazolam $(0,2 \mathrm{mg} / \mathrm{kg})$ para a manutenção do animal em um plano anestésico cirúrgico. Neste estudo, foram considerados como elevação significativa valores acima de $10 \%$ dos verificados na FC e PAS em relação aos observados no momento anterior.

Foram considerados os seguintes momentos (M) experimentais: momento zero $\left(\mathrm{M}_{0}\right)$ - animal sem anestesia; momento $1\left(\mathrm{M}_{1}\right)-15$ minutos após a medicação pré-anestésica; momento $2\left(\mathrm{M}_{2}\right)-30$ minutos após a aplicação peridural dos fármacos; momentos 3,4 e $5 \quad\left(\mathrm{M}_{3}, \mathrm{M}_{4}\right.$ e $\left.\mathrm{M}_{5}\right)$ correspondentes ao início do procedimento cirúrgico e ao pinçamento dos pedículos esquerdo e direito, respectivamente; momento 6 $\left(\mathrm{M}_{6}\right)$ - ligadura da cérvix uterina e momentos $7 \mathrm{e}$ $8\left(\mathrm{M}_{7}\right.$ e $\left.\mathrm{M}_{8}\right)$ - início da laparorrafia e fim da sutura de pele, respectivamente.

Os dados foram submetidos à análise de variância para estudar os períodos de latência e de duração da ação, e as médias comparadas por meio do teste Tukey. Os resultados foram considerados significativos quando $\mathrm{P}<0,05$ empregando-se o programa SAS/1999 (ZAR, 1998). A altura alcançada pelo bloqueio foi analisada por meio de estatística descritiva.

\section{RESULTADOS}

No grupo que recebeu a associação de ropivacaína e butorfanol, $75 \%$ dos animais permitiram a realização do procedimento cirúrgico sem necessidade de indução anestésica, enquanto no grupo da ropivacaína $100 \%$ dos animais precisaram ser induzidos à anestesia geral, devido ao estímulo doloroso.

Com relação ao período de latência dos fármacos, não foi observada diferença significativa entre os grupos quanto à perda do reflexo de esfíncter anal, da dor superficial, do tônus de cauda e do tônus postural (Tab. 1).

Com referência ao período de efeito, não se observou diferença significativa entre os grupos (Tab. 2) quanto à recuperação do reflexo do esfíncter anal, de dor superficial, do tônus de cauda, do tônus postural, da deambulação normal e do tempo de cirurgia. 
Tabela 1. Médiaterro-padrão da média do período de latência, em minutos, segundo os grupos para cada variável estudada, em cadelas

\begin{tabular}{lcc}
\hline \multicolumn{1}{c}{ Variável } & $\mathrm{G}_{1}$ & $\mathrm{G}_{2}$ \\
\hline Abolição do reflexo do esfíncter anal & $1,0 \pm 0,0$ & $1,4 \pm 0,3$ \\
Abolição da dor superficial & $3,9 \pm 0,5$ & $3,6 \pm 0,8$ \\
Abolição do tônus de cauda & $1,9 \pm 0,7$ & $1,4 \pm 0,3$ \\
Abolição do tônus postural & $3,4 \pm 0,8$ & $2,8 \pm 0,4$ \\
\hline
\end{tabular}

Diferença entre grupos não significativa pelo teste Tukey $(\mathrm{P}>0,05)$.

$\mathrm{G}_{1}$ : ropivacaína $(0,3 \mathrm{~mL} / \mathrm{kg}) ; \mathrm{G}_{2}$ : ropivacaína + butorfanol $(0,1 \mathrm{mg} / \mathrm{kg})$.

Tabela 2. Médiaterro-padrão da média da duração, em minutos, segundo os grupos para cada variável estudada, em cadelas

\begin{tabular}{lcc}
\hline \multicolumn{1}{c}{ Variável } & $\mathrm{G}_{1}$ & $\mathrm{G}_{2}$ \\
\hline Recuperação do reflexo de esfíncter anal & $319,8 \pm 28,4$ & $230,3 \pm 15,7$ \\
Recuperação da dor superficial & $375,8 \pm 54,8$ & $308,5 \pm 24,3$ \\
Recuperação do tônus de cauda & $298,0 \pm 31,1$ & $222,1 \pm 12,5$ \\
Recuperação do tônus postural & $360,8 \pm 33,8$ & $322,5 \pm 37,7$ \\
Recuperação da deambulação normal & $391,5 \pm 28,9$ & $370,8 \pm 24,7$ \\
Tempo de cirurgia & $26,0 \pm 3,0$ & $22,0 \pm 3,5$ \\
\hline
\end{tabular}

Diferença entre grupos não significativa pelo teste Tukey $(\mathrm{P}>0,05)$.

$\mathrm{G}_{1}$ : ropivacaína $(0,3 \mathrm{~mL} / \mathrm{kg}) ; \mathrm{G}_{2}$ : ropivacaína + butorfanol $(0,1 \mathrm{mg} / \mathrm{kg})$.

\section{DISCUSSÃO}

A partir de $\mathrm{M}_{4}$, todos os animais que integraram o $\mathrm{G}_{1}$ necessitaram de resgate anestésico para que se pudesse continuar a realização da cirurgia, uma vez que manifestaram sinais clínicos de dor, como elevação da frequência cardíaca e pressão arterial (Haskins, 1992; Taylor, 1995; Lascelles, 1997; Hellyer, 1998). Tal fato deveu-se, provavelmente, à progressão cranial do bloqueio obtido pela ropivacaína isolada pela via peridural, que não deve ter bloqueado a inervação dos ovários (nervo geniturinário emergente nos segmentos vertebrais $\mathrm{L}_{2}$ e $\mathrm{L}_{3}$ ).

Entretanto, em estudo mais recente, Cassu et al. (2008) utilizaram uma dose mais alta de lidocaína isolada $(8,5 \mathrm{mg} / \mathrm{kg})$ pela via peridural e, apesar do incremento na dose do anestésico local, não obtiveram êxito em $75 \%$ dos animais no que se refere à realização do procedimento cirúrgico. Tal fato, juntamente com os resultados do presente estudo, sugere que, independentemente da dose, provavelmente a utilização dos anestésicos locais de maneira isolada não alcança uma altura de bloqueio adequada para a realização de procedimentos de OSH em cadelas.

Seis animais do $G_{2}, 75 \%$, não necessitaram de resgate anestésico, o que permitiu a realização da
OSH sem necessidade de indução à anestesia geral, devido provavelmente ao bloqueio sensitivo mais cranial incrementado pela presença do butorfanol.

Silva et al. (2008) relataram progressão menos cranial do bloqueio ao administrarem ropivacaína isolada em relação aos grupos em que associaram este fármaco ao fentanil ou ao tramadol. Semelhantemente aos resultados do presente estudo, tal fato deve ter ocorrido devido à capacidade dos agentes opioides de promover certa diluição do anestésico local, o que fez com que o bloqueio se expandisse mais cranialmente, corroborando com os achados de Ishiy et al. (2002), que, ao associarem lidocaína e butorfanol, constataram bloqueio mais cranial do que com o uso do anestésico local isolado.

Além da progressão mais cranial apresentada pelo grupo que recebeu a associação do butorfanol, foi clinicamente visível a ocorrência de algum efeito sedativo, como o relatado por Camann et al. (1992) e Maia (2006), uma vez que as cadelas mantinham-se em sono profundo mesmo durante a manipulação cirúrgica, sendo que seis $(75 \%)$ apresentaram, inclusive, rotação de globo ocular.

O período de latência (3,9 minutos) encontrado no grupo da ropivacaína isolada foi semelhante 
aos resultados encontrados por Otero et al. (2003), Maia (2006) e Silva et al. (2008), que relataram $3,5, \quad 4,0$ e 3,0 minutos, respectivamente. Os resultados encontrados ao associar a ropivacaína ao butorfanol (3,6 minutos) foram diferentes dos obtidos por Maia (2006), que relatou um período de latência de 6,5 minutos. É possível que tal fato deva-se às divergências farmacogenéticas de cada animal. Lawhorn (1997), ao utilizar butorfanol associado à bupivacaína, verificou diminuição do período de latência e aumento da duração da analgesia, sem complicações para o feto em mulheres.

Para a realização de cirurgia como a OSH, faz-se necessário que a duração do bloqueio peridural seja relativamente prolongada, o que justificaria a escolha, no presente estudo, de um anestésico local de longa duração, como a ropivacaína, apesar de Feldman et al. (1996) terem constatado duração de bloqueio sensitivo de ropivacaína pela via peridural em torno de apenas 108 minutos.

Observou-se que os animais que receberam ropivacaína isolada apresentaram bloqueio sensitivo de 375,8 minutos, e o grupo que recebeu a associação do butorfanol 308,5 minutos não apresentou diferença estatística entre si. Ishiy et al. (2002), entretanto, em estudo semelhante utilizando lidocaína isolada e associada ao butorfanol, obtiveram bloqueios sensitivos mais duradouros com a adição do opioide. Sendo assim, no presente estudo, podese sugerir que a adição do agente opioide butorfanol, apesar de incrementar o bloqueio sensitivo, não interferiu na sua duração.

Feldman et al. (1996), Maia (2006) e Silva et al. (2008) relataram bloqueios menos duradouros, 133, 287,6 e 294,1 minutos, respectivamente, ao utilizarem ropivacaína . Já Maia (2006), ao associar ropivacaína e butorfanol, verificou que houve redução (239,2 minutos).

No presente estudo, foram observados períodos de duração de bloqueio sensitivo e motor superiores aos encontrados pela maioria dos autores, o que pode ser justificado pelo maior volume utilizado, semelhante ao resultado de Otero (2005), ao afirmar que a qualidade do bloqueio sensitivo e motor, ou seja, o começo da ação, a profundidade e sua duração dependerão, principalmente, da quantidade total de fármaco administrado.

\section{CONCLUSÕES}

A associação do agente opioide butorfanol incrementou a anestesia peridural com ropivacaína, promoveu bloqueio sensitivo mais cranial e menos prolongado, não interferiu de maneira significativa no período de latência e promoveu sedação pronunciada em cadelas. Sendo assim, essa associação pode ser uma opção de técnica anestésica para a realização de ovariosalpingo-histerectomia.

\section{AGRADECIMENTO}

À CAPES, pela bolsa concedida à pesquisadora.

\section{REFERÊNCIAS BIBLIOGRÁFICAS}

ALAHUHTA, S.; RASANEN, J.; JOUPPILA, P. et al. The effects of epidural ropivacaine and bupivacaine for cesarean-section on uteroplacental and fetal circulation. Anesthesiology, v.83, p.23-32, 1995.

ANDRADE, S.F.; FANTINI, D.T.; CORTOPASSI, S.R.G. et al. Terapêutica do sistema nervoso: anestésicos locais. In: ANDRADE, S.F. (Ed). Manual de terapêutica veterinária. São Paulo: Roca, 2002. cap.17, p.373-380.

CAMANN, W. R.; LOFERSKI, B. L.; FANCIULLO, G.J. et al. Does epidural administration of buthorfanol offer any clinical advantage over the intravenous route? Anesthesiology, v.72, p.216-220,1992.

CARVALHO, Y.K.; LUNA, S.P.L. Anestesia e analgesia por via epidural em cães - atualização farmacológica para uma técnica tradicional. Clin. Vet., v.12, p.68-76, 2007.

CASSU, R.N.; MENEZES, L.M.B.; KANASHIRO, C. et al. Efeitos cardiorrespiratório, analgésico e neuroendócrino da anestesia epidural com lidocaína isolada ou associada ao fentanil em cadelas submetidas à ovariosalpingohisterectomia. In: ENCONTRO DE ANESTESIOLOGIA VETERINÁRIA, 7., 2005, São Luís. Anais... São Luís, MA: CBCAV, 2005. p.174. (Resumo).

CASSU, R.N.; STEVANIN, H.; KANASHIRO, C. et al. Anestesia epidural com lidocaína isolada e associada ao fentanil para realização de ovariossalpingo-histerectomia em cadelas. Arq. Bras. Med. Vet. Zootec., v.60, p.825-831, 2008. 
ETCHES, R.C.; WRITER, W.D.R.; ANSLEY, D. et al. Continuous epidural ropivacaine $0,2 \%$ for analgesia after lower abdominal surgery. Anesth. Analg., v.84, p.784-790, 1997.

FANTONI, D.T.; KRUMENERL, J.L.J.; GALEGO, M.P. Utilização de analgésicos em pequenos animais. Clin. Vet., n.28, p.23-33, 2000.

FELDMAN, H.S.; DVOSKIN, S.; ARTHUR, G.R. et al. Antinociceptive and motor-blocking efficacy of ropivacaíne and bupivacaine after epidural administration in the dog. Reg. Anesth., v.21, p.318-325, 1996.

FREITAS, P.M.C.; EURIDES, D.; MOTA, F.C.D. et al. Estudo comparativo entre bupivacaína $0.75 \%$ e ropivacaína $0.75 \%$ em bloqueios do plexo braquial de gatos. Vet. Not., v.10, p.35-40, 2004.

GASPARINI, S.S.; LUNA, S.P.L.; CASSU, R.N. et al. Anestesia epidural com ropivacaína, lidocaína ou associação de lidocaína e xilazina em cães. Efeitos cardiorrespiratório e analgésico. Cienc. Rural, v.37, p.418-424, 2007.

HASKINS, S.C. Advantages and guidelines for using agonist opioid analgesics. Vet. Clin. N. Am.: Small Anim. Pract., v.22, p.360-361, 1992.

HELLYER, P.W.; GAYNOR, J.S. Acute postsurgical pain in dogs and cats. Compend. Contin. Educ. Pract. Vet., v.20, p.140-153, 1998.

HOUGHTON, K.J.; RECH, R.H.; SAWEYR, D.C. et al. Dose-response of intravenous butorphanol to increase visceral noceptive threshold in dogs. Soc. Exp. Biol. Med., v.197, p.290-296, 1991.

ISHY, H.M.; LUNA, S.P.L.; GONÇALVES, R.C. et al. Uso da lidocaína isolada ou associada à quetamina ou ao butorfanol, em anestesia epidural em cadelas submetidas à ovariosalpingohisterectomia. Rev. Bras. Cienc. Vet., v.9, p.134136, 2002.

LASCELLES, D.; WATERMAN, A. Analgesia in cats. In pratice, v.19, p.203-213, 1997.

LAWHORN, C.D.; STONER, J.M.; SHMITZ, M.L. et al. Caudal epidural butorphanol plus bupivacaine versus bupivacaine in pediatric outpatient genitourinary procedures. J. Clin. Anesth., v.9, p.103-108, 1997.
LUNA, S.P.L. Uso de novos fármacos no espaço epidural - aplicações clínicas. Cães Gatos, v.86, p.12, 1999.

MAIA, C.A.A. Avaliação cardiorrespiratória $e$ analgésica da ropivacaína associada à morfina, petidina ou butorfanol administrados pela via peridural em cães. 2006. 114f. Dissertação (Mestrado) - Faculdade de Medicina, Universidade Estadual Paulista, Botucatu, SP.

OTERO, P. Administração epidural e espinhal de analgésicos In: OTERO, P. (Ed). Dor - avaliação e tratamento em pequenos animais. São Paulo: Interbook, 2005. p.192-211.

OTERO, P.; TARRAGONA, L.; GUERRERO et al. Utilización de la ropivacaína al $0,2 \%$ por vía epidural en dosis única en caninos. Invet, v.5, p.5564, 2003.

PLOWMAN, A.N.; BOLSIN, S.; MATHER, L.E. Central nervous system toxicity attributable to epidural ropivacaine hydrochloride. Anaesth. Intensive Care, v.6, p.204-206, 1998.

SILVA, B.M.; MATSUBARA, L.M.; ALBUQUERQUE, V.B. et al. Ropivacaína isolada e associada ao fentanil ou ao tramadol administrados pela via peridural em cães. Cienc. Rural, v.38, p.2197-2202, 2008.

TAYLOR, R.; McGEHEE, R. Manual of small animal postoperative care. Baltimore: Williams \& Wilkins, 1995. p.74-81.

TSANG, B.K.; HE, Z.; WONGCHANAPAI, W. et al. Visceral analgesic tolerance to intratecal butorfanol in rats. Can. J. Anesth., v.45, p.10191023, 1998.

WEBSTER, C.R.L. Opioides In: WEBSTER, C.R.L. (Ed). Farmacologia clínica em medicina veterinária. São Paulo: Roca, 2005. cap.30, p.6061.

ZAR, J.H. Biostatistical analysis. 4.ed. New Jersey: Prentice-Hall, 1998. 930p. 